Cahiers $d u$ MONDE RUSSE

\section{Cahiers du monde russe}

Russie - Empire russe - Union soviétique et États indépendants

48/2-3 | 2007

Les résonances de 1905

\title{
Le culte russe de la Révolution française
}

\section{Alexandre V. Tchoudinov}

\section{OpenEdition \\ Journals}

Édition électronique

URL : https://journals.openedition.org/monderusse/9022

DOI : 10.4000/monderusse. 9022

ISSN : $1777-5388$

\section{Éditeur}

Éditions de l'EHESS

\section{Édition imprimée}

Date de publication : 15 avril 2007

Pagination : 485-498

ISBN : 978-2-7132-2147-7

ISSN : $1252-6576$

\section{Référence électronique}

Alexandre V. Tchoudinov, "Le culte russe de la Révolution française ", Cahiers du monde russe [En ligne], 48/2-3 | 2007, mis en ligne le 01 janvier 2007, consulté le 02 septembre 2022. URL : http:// journals.openedition.org/monderusse/9022 ; DOI : https://doi.org/10.4000/monderusse. 9022 
chercher : repérer : avancer

Cet article est disponible en ligne à l'adresse :

http://www.cairn.info/article.php?ID REVUE=CMR\&ID NUMPUBLIE=CMR 482\&ID ARTICLE=CMR 4820485

\section{Le culte russe de la Révolution française}

par Alexandre V. TCHOUDINOV

\section{Editions de l'EHESS | Cahiers du monde russe}

2007/2-3 - Vol 48

ISSN 1252-6576 | ISBN 9782713221477 | pages 485 à 498

Pour citer cet article :

-Tchoudinov A., Le culte russe de la Révolution française, Cahiers du monde russe 2007/ 2-3, Vol 48, p. 485-498.

Distribution électronique Cairn pour les Editions de l'EHESS.

(C) Editions de l'EHESS. Tous droits réservés pour tous pays.

La reproduction ou représentation de cet article, notamment par photocopie, n'est autorisée que dans les limites des conditions générales d'utilisation du site ou, le cas échéant, des conditions générales de la licence souscrite par votre établissement. Toute autre reproduction ou représentation, en tout ou partie, sous quelque forme et de quelque manière que ce soit, est interdite sauf accord préalable et écrit de l'éditeur, en dehors des cas prévus par la législation en vigueur en France. Il est précisé que son stockage dans une base de données est également interdit. 


\section{LE CULTE RUSSE DE LA RÉVOLUTION FRANÇAISE}

De tous les événements historiques survenus à l'étranger, c'est sans aucun doute la Révolution française qui a le plus influencé la conscience culturelle et politique de la Russie. Son influence sur les idées et pratiques politiques russes a été bien étudiée ${ }^{1}$. Dès le XIX ${ }^{\mathrm{e}}$ siècle, l'image de la Révolution française constitua non seulement un phénomène de la culture politique, mais aussi une partie importante de la mémoire historique de l'intelligentsia russe. Nous nous proposons d'examiner ciaprès ce sujet qui n'est pas encore assez éclairci dans l'historiographie.

Par «mémoire historique », nous entendons ici le sens donné par Pierre Nora aux « Lieux de mémoires », opposant les notions de « mémoire » et d' « histoire » :

Mémoire, histoire : loin d'être synonymes, nous prenons consience que tout les oppose. La mémoire est la vie, toujours portée par des groupes vivants et, à ce titre, elle est en évolution permanente, ouverte à la dialectique du souvenir et de l'amnésie, inconsciente de ses déformations successives, vulnérable à toutes les utilisations et manipulations, susceptible de longues latences et de soudaines revitalisations. L'histoire est la reconstruction toujours problématique et incomplète de ce qui n'est plus. La mémoire est un phénomène toujours actuel, un lien vécu au présent éternel ; l'histoire, une representation du passé. Parce qu'elle est affective et magique, la mémoire ne s'accommode que des détails qui la confortent ; elle se nourrit de souvenirs flous, télescopants, globaux ou flottants, particuliers ou symboliques, sensible à tous les transferts, écrans, censure ou projections. L'histoire, parce que opération intellectuelle et laïcisante, appelle analyse et discours critique. La mémoire installe le souvenir dans le sacré, l'histoire l'en débusque, elle prosaïse toujours. La mémoire sourd d'un groupe

1. Voir Tamara Kondratieva, Bolcheviks et Jacobins : itinéraire des analogies, P. : 1989 ; Dmitry Shlapentokh, The French Revolution in Russian intellectual life. 1865-1905, Wesport (Conn.) - Londres : Praeger, 1996 ; Idem, The French Revolution and Russian anti-democratic tradition. A case of false consciousness, New Brunswick - Londres: Transactions Publishers, 1997 ; A.V. Gordon, «Velikaja francuzskaja revoljucija kak javlenie russkoj kul'tury (k postanovke voprosa) », in A.V. Čudinov [Tchoudinov], éd., Istoričeskie etjudy o Francuzskoj revoljucii. Pamjati V.M. Dalina, M., 1998. 
qu'elle soude, ce qui revient à dire, comme Halbwachs l'a fait, qu'il y a autant de mémoires que des groupes; qu'elle est, par nature, multiple et démultipliée, collective, plurielle et individualisée. L'histoire, au contraire, appartient à tous et à personne, ce qui lui donne vocation à l'universel².

La vision de la Révolution française, qui s'est construite dans la culture russe du $\mathrm{XIX}^{\mathrm{e}}$ siècle, appartenait en effet plus à la sphère du sacré qu'au domaine de la connaissance scientifique. De plus, il est important de souligner que le processus même de formation de cette vision procédait par l'omission consciente de certaines réalités historiques, celles qui contredisaient la représentation idéalisée de la Révolution.

Cette métamorphose a été progressive. Les contemporains directs et surtout les témoins oculaires de la Révolution, dont elle avait marqué le destin, étaient fort éloignés de l'idée de la transformer en objet d'adoration. Les exemples sont nombreux, de personnes élevées dans les valeurs de la culture des Lumières qui, dans un premier temps, ont considéré les débuts de la Révolution comme la réalisation des idéaux humanistes et qui, ensuite, ont éprouvé un véritable choc en constatant à quel point la réalité allait à l'encontre de leurs attentes. C'est notamment le cas de N.I. Karamzin qui, au cours de son séjour à Paris en 1790, a observé non sans sympathie le déroulement des événements encore pacifiques de cette première période de la Révolution. Cependant cinq ans plus tard, il écrivait ces lignes connues, que A.I. Herzen qualifiera « de larmes et de feu » : «Siècle des Lumières ! Je ne te reconnais pas. Dans le sang et les flammes, je ne te reconnais pas. Au milieu des meurtres et des destructions, je ne te reconnais pas $! »^{3}$. De même à la fin du XVIII ${ }^{\mathrm{e}}$ siècle, la vision du monde de A.N. Radičev n'est pas moins tragique :

Le bonheur, la vertu et la liberté ont été dévorés par le gouffre furieux

Vois, les débris horribles surnagent encore.

Non, tu ne seras pas oublié, siècle fou et sage,

Tu seras maudit pour toujours, à la surprise de tous. ${ }^{4}$

Telle était donc la réaction de ceux qui avaient vécu directement la Révolution française, et pour qui elle était devenue un drame personnel. En revanche, leurs contemporains plus jeunes, ceux de la génération suivante, éprouvaient déjà de tout autres sentiments. Pour eux, la Révolution était récente, mais elle appartenait malgré tout au passé. La manière dont elle était perçue par ces jeunes gens, opposés à la réalité russe, est parfaitement exprimée par la célèbre phrase d'Alexandre I : « Dans la Révolution française, il faut distinguer les principes et les crimes $»^{5}$. Une telle

2. Pierre Nora « Entre Mémoire et Histoire », in Les Lieux de mémoire, Pierre Nora, éd., t. 1. P. : Gallimard, 1984, p. XIX.

3. Nikolaj Mihajlovič Karamzin, «Melodor k Filaletu», in Izbrannye pis'ma i stat'i, N.M. Karamzin, M. : Sovremennik, 1982, p. 148-149.

4. Aleksandre Nikolaevič Radiščev, «Osmnadcatoe stoletie », in Izbrannoe, A.N. Radiščev, M. : Moskovskij rabočij, 1959. p. 260.

5. D’après Evgenij Grigor'evič Plimak et Vladimir Georgievič Horos, « La Révolution française et la tradition révolutionnaire en Russie » in La Révolution française et la Russie, M. : Ed. du Progrès, 1989, p. 217. 
manière de voir, supposait une approche sélective de l'expérience de la Révolution, en divisant son histoire entre ce qui est acceptable, et ce qui est inadmissible. C'est précisément cette position que les décembristes en particulier ont adoptée, puisqu'ils «détestaient les crimes et appréciaient les règles de la Révolution française $»^{6}$. Le récit des événements réels allait jusqu'à provoquer leur irritation, car ceux-ci étaient perçus comme la condamnation implicite et le démenti de l'idéal. En lisant les Lettres d'un voyageur russe de N.M. Karamzin, Nikita Murav'ev a griffonné quelques remarques dans la marge du livre, en face de passages où l'auteur donnait ses impressions sur le Paris révolutionnaire : «C'est si stupide, que je ne trouve rien à dire », «c'est faux », « imbécile ». La remarque de Murav'ev en face de la phrase de Karamzin qui termine le passage sur Condorcet, est particulièrement significative. En effet, face à cette phrase qu'écrit Karamzin : « le peuple est la lame aiguisée, avec laquelle il est dangereux de jouer, et la révolution, le cercueil ouvert de la vertu, et le Mal personifié », Murav'ev note : «Tout ceci est probablement vrai. Mais la morale est mauvaise $\gg^{7}$. Sa remarque montre qu'il n'a rien à objecter au fond, mais que personne n'a envie d'y croire.

Cependant en divisant l'image de la Révolution en deux parts égales et indépendantes l'une de l'autre, d'un côté le « bel » idéal, de l'autre la «mauvaise » réalité, les décembristes ne négligeaient en aucun cas ce second aspect. Ils se donnaient en toute conscience pour but de ne pas permettre que se répètent « les événements horribles, qui se déroulèrent en France pendant la révolution $»^{8}$. Ils étaient ainsi loin de s'identifier aux hommes de la Révolution française, qui de gré ou de force, formaient cette réalité « inacceptable», et en portaient la responsabilité.

La perception de la génération suivante des intellectuels d'opposition issus de milieux roturiers dans les années 1840 avait déjà évolué. Pour eux, la vision idéale de la Révolution française avait pris une signification dominante, reléguant peu à peu au second plan son récit réel. Contrairement aux décembristes, les membres des cercles d'opposition, qui luttaient uniquement par la parole contre la réalité sociale qui les entourait, n'avaient pas de raison d'attendre, ni avec espoir ni avec circonspection, la répétition des événements révolutionnaires sur le sol russe dans un futur proche. En conséquence, ils ne voyaient dans la Révolution française qu'un principe abstrait, le symbole de la négation, du renversement. «La négation est mon Dieu », écrivait V.G. Belinskij à un ami en 1841. «Dans l'histoire, mes héros sont

6. Citation de M.F. Orlov. Voir Mitrofan Viktorovič Dovnar-Zapol'skij, Memuary dekabristov, Kiev: Ivanov, 1906. p. 5. Voici aussi l'opinion de P.G. Kahovskij : «La Révolution en France, commencée de manière si bienfaisante, est malheureusement passée sur la fin du domaine du légal au domaine du criminel », cité dans S.S. Volk, Istoričeskie vzgljady dekabristov, M.-L., 1958. p. 264. Pour plus de détails, voir aussi Anna Vladimirovna Semenova, «Dekabristy i Francuzskaja revoljucija konca XVIII v. », in Novaja i novejšaja istorija, 1989. t. 3 ; S.A. Ekšut, «Velikaja francuzskaja revoljucija i nravstvennye iskanija dekabristov », in Naučnye doklady vyščej školy filos. nauki, 1990, t. 1 ; ou encore Vadim Surenovič Parsamov, Dekabristy i francuzskij liberalism, M. : Polimed, 2001.

7. Voir Jurij Mihajlovič Lotman, Sotvorenie Karamzina, M. : Molodaja gvardija, 1998. p. 375, ex. 21 .

8. Vosstanie dekabristov. M.-L., 1927.t. 4, p. 90. 
les démolisseurs de l'ordre ancien, Luther, Voltaire, les Encyclopédistes, les terroristes, Byron... $»^{9}$. La plupart des personnes de l'entourage de Belinskij, comme I.I. Panaev, V.P. Botkin, A.I. Herzen et d'autres, éprouvait la même admiration pour les «terroristes » Robespierre et Saint-Just. Leurs adversaires, qui ne partageaient pas ce culte pour les Jacobins, tel T.N. Granovskij, s'enthousiasmaient pour d'autres républicains révolutionnaires, comme les Girondins.

La référence à Dieu, dans la citation de Belinskij, est certainement plus qu'une simple métaphore. L'adoration vouée à la Révolution française, comme réalisation du principe de négation de l'ordre ancien, acquit effectivement le caractère d'un culte original, à propos duquel Herzen écrivit plus tard : « Le culte de la Révolution française, c'est la première religion d'un jeune Russe ; et qui de nous ne possédait pas, en cachette, les portraits de Danton et de Robespierre $»^{10}$.

Ce culte de la Révolution était principalement conditionné par le fait que l'intelligentsia russe puisait une grande part de ses informations dans les travaux des historiens français libéraux et socialistes de la Restauration et de la Monarchie de juillet. Ce sont eux qui, en vertu de la conjoncture politique et idéologique qui existait alors en France, ont jeté les bases de cette interprétation idéalisée des événements révolutionnaires du XVIII ${ }^{\mathrm{e}}$ siècle, qui au XXe siècle, sous la plume de l'historien anglais A. Cobban, a reçu le nom de «mythe de la Révolution française » ${ }^{11}$. Toutefois les intellectuels russes ne se posaient pas la question du bien-fondé scientifique de leur interprétation : premièrement, il n'y avait pas encore en Russie d'historiens de la Révolution française capables de porter à ce sujet le moindre jugement professionnel et, deuxièmement, la position idéologique de chaque auteur avait une signification bien plus importante. Ainsi l'argument avancé par Botkin contre Granovskij est particulièrement caractéristique : «Son opinion à propos de Robespierre et des Girondins est absolument opposée aux avis de tous les meilleurs cerveaux de France, et en particulier à celui de Leroux $»^{12}$.

Les membres des cercles radicaux des années 1840, non seulement admiraient les révolutionnaires français, mais aussi s'identifiaient directement à eux, se divisant ainsi en « jacobins » et « girondins » dans leurs débats sur la Révolution. Dans ce monde des idées, ce monde des mots, grâce auquel ils se protégeaient de la réalité jugée inacceptable, et dans lequel ils vivaient de manière originale, la Révolution française idéalisée restait pour eux le présent éternel.

9. Vissarion Grigor'evič Belinskij, Sobranie sočinenij, M. : Hudožestvennaja literatura, 1982, t. 9 , p. 483.

10. Aleksandr Ivanovič Herzen, «Au citoyen rédacteur de 1"Homme" », in A.I. Gercen, Sobranie sočinenij, M. : Izdat. Akadem. Nauk SSSR, 1963. t. 30, livre 2, p. 502 (original en français).

11. Voir Alfred Cobban, « The Myth of the French Revolution (1954) », in A. Cobban, ed., Aspects of the French Revolution, Londres : Cape, 1968.

12. V.G. Belinskij, Pis'ma v 3-h tomah, SPb. : Stasjulevič, 1914, t. 2, p. 424. Pierre Leroux (1797-1871) est un saint-simonien, dont les travaux ont connu une grande popularité dans les cercles radicaux des années 1840 . 
Certes, il ne faut pas en déduire, que ces intellectuels russes étaient prêts à répandre le sang dans la vie réelle, tout comme les héros qu'ils admiraient. Les membres des cercles des années 1840, qui ont vécu jusqu'à la limite des années 1850 et 1860 , alors qu'une réelle menace pesait de voir la révolution passer subitement du monde de l'abstrait à la réalité, ont condamné les appels à la violence, à la « hache », des représentants les plus radicaux de la nouvelle génération des intellectuels d'opposition ${ }^{13}$.

Dans l'historiographie existante, la question de l'influence de l'expérience de la Révolution française dans les différents courants révolutionnaires des années 18601870, a été examinée à maintes reprises ${ }^{14}$. En revanche les études ont plus rarement abordé le thème de sa perception par les couches plus larges de l'intelligentsia, qui n'étaient pas liées directement à l'agitation clandestine révolutionnaire, mais qui cependant étaient pénétrées à des degrés divers par des humeurs d'oppositions, par des volontés confuses de changement. Pourtant, c'est justement dans ce milieu, qui n'était pas confronté au problème de faire correspondre ses activités pratiques personnelles avec l'expérience des révolutionnaires du XVIII ${ }^{\mathrm{e}}$ siècle, que la vision mythologique de la Révolution française a connu sa plus large diffusion. Les sentiments éprouvés à son égard devinrent une sorte de code, par lequel se reconnaissaient les membres de ce groupe social, ceux qui avaient une «éducation moderne », les progressistes. C'est ainsi que des années plus tard, le professeur de physique de l'université de Moscou N.A. Ljubimov se souvenait de cette période :

Quelle jouissance procurait un fruit défendu, acquis par quelque professeur ou autre chanceux : tenir entre ses mains, même pour un court moment, un volume dangereux de quelque histoire de la révolution, dans lequel, semblait-il, se trouvait justement la vérité la plus cachée. On se rappelle avec quelle avidité, nous dévorions en une nuit un tome de Michelet ou de Louis Blanc de quatre ou cinq cents pages [...] Nos passions de jeunesse furent favorisées par ce que dans les cercles littéraires ou professoraux, qui avaient le plus d'influence sur les jeunes esprits, ce $[\ldots]$ «culte de la révolution », [...] faisait partie de nos convictions élémentaires, sinon dans les détails de la réalisation de son programme, du moins dans ses principes, dans ses idées, qui devaient impérativement se concrétiser et séparer le nouveau monde de l'ancien. Les adeptes français modérés du culte de la révolution nomment cela les principes immortels de 1789 . Nous en comprenions le sens de manière plus vague, mais plus large, considérant la révolution comme un aspect fondamental de la notion générale de progrès historique. [...] Pour la majorité de ceux qui se rangeaient alors parmi les modernes, ces idées traversaient l'esprit comme un nuage léger et flou. Le culte de la révolution apparaissait sous forme d'une adoration éloignée, il était sans importance pratique ni force. ${ }^{15}$

13. Pour plus de détails, voir Kondratieva, Bolcheviks et Jacobins...

14. Voir par exemple Ibid., p. 34-37, ainsi que Plimak, Horos, Velikaja francuzskaja revoljucia...., p. 245-249.

15. V. Kočnev, [N.A. Ljubimov], «Protiv tečenija. Besedy o revoljucii. Nabroski i očerki v razgovorah dvuh prijatelej », Russkij vestnik, août 1880, p. 613-617. 
Ce témoignage est d'autant plus précieux que son auteur, dans un âge plus avancé, adopta une position extrêmement conservatrice et anti-révolutionnaire. Cependant ses souvenirs correspondent bien à ceux d'un autre contemporain au destin tout à fait différent, V.K. Debogorij-Mokrievič, passé quant à lui du même cercle d'étudiants affranchis au camp des révolutionnaires :

Sur l'histoire de la Révolution française, les ouvrages qui étaient principalement traduits et vulgarisés étaient ceux des auteurs dithyrambiques. Nous nous en délections. Nous connaissions sur le bout des doigts les noms de tous les héros de la Révolution française, depuis les principaux meneurs jusqu'aux individus de second, et même de troisième plan. Aux uns plaisait Danton, aux autres Camille Desmoulins, tandis que d'autres enfin rêvaient de Saint-Just. Telle était l'atmosphère dans laquelle nous baignions dans les années 70, attisant en nousmêmes la flamme révolutionnaire ${ }^{16}$.

La révolution était non seulement désirée, mais aussi considérée comme inévitable. Non pas une révolution semblable à celle qui s'était produite en France, qui avait eu lieu dans la réalité, avec les massacres de septembre, la guerre civile en Vendée, les noyades de Nantes, la Grande Terreur, etc., mais cette «Révolution française » dont l'image idéalisée avait vécu de génération en génération dans la mémoire historique de l'intelligentsia russe. N.I. Kareev se souvenait, que «quiconque commençait sa vie consciente dans les années soixante-soixante-dix du siècle passé, ne pouvait pas ne pas réfléchir au moment et à la façon dont la longue révolution de l'Europe de l'Ouest, dans son flux irrésistible, s'emparerait de la Russie, elle qui avait déjà commencé dès l'époque des décembristes à exercer son influence sur les cercles avancés de notre société ${ }^{17}$.

Cette signification bien particulière, presque sacrée, donnée à la Révolution française par ces gens «progressistes », trouve encore un témoignage avec l'utilisation de l'épithète «grande», dans le sens d'illustre, dans l'expression «la Grande Révolution française ». La question de savoir par qui, quand et dans quel contexte ce qualificatif a été utilisé pour la première fois mérite une étude à part. Cependant, le fait est que dès la fin du XIX siècle, dans l'historiographie russe, les termes «Grande Révolution française » sont employés comme une expression consacrée, ce qui n'a d'équivalent dans aucun autre pays.

L'étude professionnelle de la Révolution française, qui a débuté en Russie après les grandes réformes, non seulement n'a pas détruit ce « culte », mais elle n'a fait que l'affermir davantage. Ceci n'a rien d'étonnant, dans la mesure où les grands historiens de « l'école russe », à quelques rares exceptions près, appartenaient à ce même groupe social «progressiste», ou «moderne» de l'intelligentsia, pour laquelle le culte de la Révolution française faisait office d'un moyen d'auto-identification et de rassemblement. Partageant les convictions et les erreurs de leur

16. V.K. Debogorij-Mokrievič, « Avtobiografija », Dejateli SSSR i revoljucionnogo dviženija Rossii. Enciklopedičeskij slovar' Granat, M., 1989. Koll. 89.

17. Nikolaj Ivanovič Kareev, Prožitoe i perežitoe, L.: Izdat. leningradskij universitet, 1990, p. 289. 
milieu, en proie au diktat de la mémoire collective, les historiens, de gré ou de force, implicitement ou explicitement, reproduisaient dans leurs travaux ce même mythe, lui donnant cette confirmation « scientifique » attendue par leurs lecteurs.

Il ne faut cependant en aucune manière en déduire que les historiens de « l'école russe » altéraient ou falsifiaient les faits. Ils menaient leurs recherches sur des problèmes concrets à un niveau professionnel très élevé et, dans de nombreux cas, leurs études n'ont rien perdu de leur valeur scientifique. D'un autre côté, les travaux généraux, destinés au grand public, montrent la Révolution française sous un seul angle, comme une sorte de fête, personnifiant la joie de la victoire de la liberté sur le despotisme. Les faits n'étaient pas déformés, mais tout simplement passés sous silence. Les aspects sombres de la Révolution étaient dissimulés et la question de son « coût » était laissée de côté.

À ce sujet, il est intéressant de comparer deux œuvres de E.V. Tarlé, un des éminents représentants de «l'école russe»: son travail de publiciste intitulé La chute de l'absolutisme dans l'Europe de l'Ouest, publié en 1906, au moment de la première révolution russe et sa thèse fondamentale La classe ouvrière en France à l'époque de la Révolution, publiée en 1909-1911. Dans le premier ouvrage, en comparant la France de la fin du XVIII siècle à la Russie du début du XXe siècle, Tarlé pousse instamment le lecteur à conclure que la monarchie russe, tout comme la monarchie française de l'époque, doit inévitablement tomber à la suite d'une révolution. Le sens des larges raisonnements de l'historien est en réalité assez simple : si l'absolutisme est « un désastre » aussi bien dans la sphère économique ${ }^{18}$, que dans la sphère politique ${ }^{19}$, alors la révolution qui le détruit est indiscutablement bénéfique. En même temps, pratiquement tous les coûts sociaux et économiques de la Révolution française sont passés sous silence. De tous ses « excès », seul l'épisode de la tête du commandant de la Bastille promenée sur une pique est mentionné. On ne trouve pas un mot en revanche au sujet des nombreux actes de violence massive perpétrés par la foule, ni sur la grande Terreur. Le thème de la Vendée, n'ayant semble-t-il pas le moindre rapport avec la réalité russe, est résumé en une seule et unique phrase : « Notre absolutisme n'avait pas et ne pouvait pas avoir de Vendée, parce qu'on ne peut pas inventer la Vendée comme les Centuries noires $»^{20}$.

Dans la thèse en revanche, en suivant scrupuleusement les faits, les documents, Tarlé parle assez ouvertement du coût des transformations révolutionnaires, et dessine un tableau vraiment très sombre de la vie quotidienne des Français, et surtout des ouvriers, pendant la Révolution. En outre, il reconnaît sincèrement, que la désorganisation temporaire de la vie productive, liée inévitablement à la guerre et aux désordres intérieurs, mais aussi et surtout la politique même du gouvernement

18. «Le désastre économique », «l'obstacle au développement naturel de la vie économique du pays », etc. Voir Evgenij Viktorovič Tarle, «Padenie absoljutizma v Zapadnoj Evrope. Istoričeskie očerki », in E.V. Tarle, Sočinenija v 12-ti tomah, M. : Izdat. Akad. nauk SSSR, 1958 , t. 4, p. 328, 338, 393 et suivantes.

19. Ibid., p. 355

20. Ibid., p. 439-440. 
révolutionnaire, étaient responsables de la dure crise économique et sociale, que vivait alors la France : la loi du «maximum», les réquisitions, la Terreur ${ }^{21}$. Il est vrai qu'à la différence du premier ouvrage, destiné au grand public, la thèse était accessible seulement à un cercle étroit de spécialistes.

De tels contrastes dans l'éclairage de la Révolution, chez un seul et même auteur, à travers des œuvres destinées à des publics différents, ne sont certes pas un hasard. D'autres historiens libéraux de l'époque préféraient eux aussi laisser de côté les faits « dérangeants », susceptibles de faire de l'ombre à l'image idéalisée de la Révolution, dans leurs travaux publiés dans le but d'obtenir une plus ou moins large résonance publique. Le maître de «l'école russe » Kareev évoque sans détour dans ses mémoires le choix tout à fait conscient d'un tel raccourci historique :

D'après la vieille tradition, édifiée sur les histoires de la révolution les plus anciennes (Mignet et Thiers, Michelet et Louis Blanc), par ses anciens adulateurs, c'est avant tout le côté représentatif, héroïque et festif de la révolution, devenue une légende poétique, qui sautait au yeux. Le serment du Jeu de paume, la prise de la Bastille, la nuit du 4 août, la fête de la Fédération, la Déclaration des droits de l'homme, la Marseillaise, sont en effet de beaux moments, impressionnants, capables d'enthousiasmer au plus haut point. Mais tout cela est justement le côté poétique, festif et représentatif de la Révolution, qui avait aussi sa prose, son quotidien, son envers, et à côté de l'héroïsme, sa pathologie.22

Bien que les historiens fussent au courant de cette « prose » et de cette « pathologie », ils écrivaient malgré tout essentiellement sur le côté « représentatif ». Cela n'a finalement rien d'étonnant. Ils aspiraient à apporter leur contribution au mouvement socialpolitique pour la rénovation de la société russe. Ainsi dans leurs interventions adressées à un large public, ils interprétaient la Révolution française, moins comme un événement réel $d u$ passé, que comme la personnification de l'idéal libéral, dont ils rêvaient qu'il deviendrait le futur de la Russie. Kareev se souvenait par exemple de son activité politique lors de la première révolution russe : «Dans les meetings et dans les réunions électorales, je suis intervenu très souvent dans les salles les plus diverses, exposant habituellement les principes fondamentaux du parti [des constitutionnels démocrates], que je réduisais surtout aux idées de la Déclaration des droits de l'homme et du citoyen des temps de la Révolution française... »23.

Et si les historiens professionnels eux-mêmes n'étaient pas assez impartiaux dans leur éclairage des événements français de la fin du XVIII siècle, que dire alors des publicistes et des vulgarisateurs, qui ont activement abordé ce sujet pendant et après la révolution en 1905-1907. Le récit de la Révolution française leur servait de prétexte pour exprimer, plus ou moins directement, leur attitude négative par rapport à la réalité russe, et appeler à son changement par la voie révolutionnaire. En

21. E.V. Tarle, « Rabočij klass vo Francii v epoku Revoljucii », in Tarle, éd., Sočinenija. t. 2, chapitre 5 .

22. Kareev, Prožitoe i perežitoe, p. 289.

23. Ibid., p. 235. 
conséquence, ces auteurs recherchaient la ressemblance entre la France de l'Ancien Régime et la Russie du XIX et du début du XX⿳亠丷厂犬 siècle. Ainsi, ils identifiaient obstinément la monarchie des Bourbons avec l'autocratie russe, et parfois même ils attribuaient aux paysans français la foi bien russe en korol'-batjuška [le roi père] ${ }^{24}$. Les paysans français eux-mêmes étaient d'ailleurs représentés comme des serfs à la russe c'est-à-dire à peu près comme des esclaves ${ }^{25}$. Cela n'était en aucun cas la réalité, mais au moins cette image permettait de donner à la France prérévolutionnaire des caractéristiques facilement reconnaissables pour les lecteurs russes.

Par ailleurs, ces ouvrages cherchaient souvent à arrondir les angles de l'histoire révolutionnaire, à « retoucher» les événements qui n'étaient pas en accord avec l'image de la Révolution comme une « fête de la Liberté ». L'un de ces événements tristement célèbres est le massacre de septembre 1792 dans les prisons, lorsque des centaines de prisonniers, dont la grande majorité n'avaient rien à voir avec la politique, furent mis en pièces par la foule. Cet épisode tragique était ainsi représenté dans les livres populaires sur la Révolution :

À partir du 2 septembre, le peuple en foule commença à faire la tournée des prisons et à tuer les prisonniers. On ne les tuait pas tous, mais d'après une liste, préparée d'avance et contrôlée ; ceux qui n'étaient pas dans cette liste étaient remis en liberté ${ }^{26}$.

[...]le jour de l'engagement des volontaires se transforma en jour de justice sommaire populaire, en jour de massacres sanglants des monarchistes, des ennemis intérieurs de la patrie [...] Dans une des prisons, à l'Abbaye, on tenta d'instaurer quelque chose comme un tribunal. Les prisonniers subissaient un interrogatoire. Immédiatement après on rendait le verdict de condamnation ou d'acquittement [...] Les Parisiens tuaient leurs ennemis pour protéger leurs proches ${ }^{27}$.

Sous cet angle, le lecteur avait l'impression que cette violence "contrôlée » s'appliquait uniquement à des individus effectivement coupables. Sous la plume d'un auteur anonyme d'une brochure sociale-démocrate consacrée à la Révolution française, qui a été rééditée par les Bolcheviks à plusieurs reprises après 1917, le massacre de septembre devient un procès judiciaire bien réglé contre les criminels :

[...] À cette époque-là, de nombreux aristocrates et prêtres étaient enfermés dans les prisons de la ville. C'est contre eux que se dressa la population parisienne. Le

24. N. Nikolin, Leonid Nikolaevič Andreev, Velikij perevorot ili Velikaja francuzskaja revoljucija, SPb., 1908. Partie 2, p. 3.

25. Ibid., 1 $1^{\text {re }}$ partie, SPb., 1907, p. 10-12 ; Voir aussi M. Olenina, Vesna narodov (Velikaja francuzskaja revoljucija), Nijni-Novgorod, 1906, p. 10, 75-76; E. Efrusi, Velikaja revoljucija vo Francii, M., 1908., p. 3-4. De nombreux travaux de publicistes ou de vulgarisateurs publiés en Russie peu après la révolution de 1917, présentent aussi la condition des paysans dans la France pré-révolutionnaire comme servile. Voir par exemple, A.I. Sražev, Kak Francuzy dobyli i poterjali svoju svobodu (Iz istorii Velikoj revoljucii), M., 1917. p. 7-8 ; O.A. Vol’kenštejn, Velikaja francuzskaja revoljucija 1789 g., M., [1917], p. 6, 19 ; T.A. Bogdanovič, Velikaja francuzskaja revoljucija,L.-M., 1925. p. 5, 195.

26. Nikolin, Andreev, Velikij perevorot...., 2 $2^{\mathrm{e}}$ partie, p. 72.

27. Efrusi, Velikaja revoljucija., p. 66-67. Souligné par nous. 
2 septembre, une foule fit irruption dans ces prisons, et commença à tuer les criminels. Au début, on tua sans aucun jugement, mais au bout de quelques heures, un tribunal populaire fut instauré, qui examinait d'abord les crimes des accusés, écoutait ensuite leur défense, et seulement après prononçait son arrêt. De cette manière, nombreux sont ceux qui ont évité la mort. ${ }^{28}$

De même, la Terreur de 1793-1794 était représentée comme dirigée uniquement contre les ennemis de la Révolution :

Les révoltés contre la République, les aristocrates, étaient traités avec toute la sévérité possible. Une telle politique est appelée Terreur, c'est-à-dire intimidation. [...] Si la Révolution n'avait pas châtié ses ennemis, elle serait elle-même morte ».

Ce n'est en revanche pas le cas pour Thermidor :

Sous le gouvernement bourgeois, des gens innocents moururent en masse, des gens sincèrement dévoués à la révolution, tandis qu'au cours des années précédentes n'étaient morts que les traîtres et les individus suspects, dans tous les cas des ennemis du peuple..$^{29}$

Même les auteurs qui n'éprouvaient pas de sympathie particulière à l'égard de la Terreur, comme l'auteur anonyme de la brochure sociale-démocrate citée cidessus, justifiaient celle-ci par les circonstances de la guerre :

La cruauté et l'absence de pitié du gouvernement républicain à l'égard des ennemis intérieurs ne peut s'expliquer que par le contexte tendu dans lequel vivaient alors les Français. ${ }^{30}$

Malgré toute l'horreur des mesures terroristes, la grande majorité du peuple soutenait le gouvernement, car elle voyait qu'il n'y avait pas d'autre possibilité pour conserver l'unité et la solidité de la République. ${ }^{31}$

À cela, il faut ajouter encore une page douloureuse de l'histoire de France de la fin du XVIII siècle : la guerre civile en Vendée. Au cours de cet épisode, les troupes révolutionnaires, après avoir écrasé dans une lutte acharnée les paysans révoltés, se livrèrent à de véritables massacres contre la population civile et ce, sur les ordres du pouvoir révolutionnaire. Dans la représentation de l'histoire de la Révolution française popularisée en Russie, ces événements relevaient uniquement d'actes de guerre, et les atrocités étaient partagées de part et d'autre :

La guerre était menée avec une cruauté terrible. Les prisonniers, tombés aux mains des Vendéens, étaient immédiatement fusillés. Les prisonniers vendéens

28. Velikaja francuzskaja revoljucija, SPb., 1906. p. 34. (Il existe de nombreuses rééditions de cette brochure : M., 1917 ; M.-Petrograd, 1918 ; Gomel', 1919, etc.). C'est nous qui soulignons.

29. Ibid., p. 39, 42. C'est nous qui soulignons.

30. Efrusi, Velikaja revoljucija...,p. 97.

31. Nikolin, Andreev, Velikij perevorot..., 2 ${ }^{\mathrm{e}}$ partie, p. 95. 
eux aussi subissaient le même sort. Après de nombreux accrochages et affrontements sanglants, que nous ne raconterons pas ici, l'insurrection en Vendée fut réprimée. Cela se passa en décembre $1793[\ldots]^{32}$

Le fait que cet auteur termine son récit sur la Vendée justement en décembre 1793 est particulièrement significatif. Cela lui évite d'évoquer, après la destruction des forces armées vendéennes, au début du mois de janvier 1794, la marche punitive des «colonnes infernales » républicaines contre la population civile du département. En effet, ces derniers faits auraient sans aucun doute assombri l'image lumineuse de la Révolution développée tout au long de l'ouvrage. Il faut souligner qu'il n'y a aucun mensonge dans ce passage, à la différence de l'affirmation absurde concernant les listes de septembre «préparées d'avance et contrôlées ». Le fait que les prisonniers étaient «immédiatement» fusillés est une légère exagération puisque, en réalité, ils subissaient fréquemment les tortures les plus atroces. Cependant si l'on excepte cette petite imprécision, tout le reste du récit est la pure vérité, mais seulement très sélective, au point que l'histoire du carnage de la population rebelle du département par le pouvoir révolutionnaire est finalement représentée par le littérateur comme un épisode ordinaire des hostilités, même s’il reconnaît qu'il est particulièrement féroce.

Dans les ouvrages cités, il est possible de trouver un exemple encore plus probant de l'utilisation «virtuose» des matériaux. Un des auteurs, sans aller jusqu'à recourir à la falsification ouverte, alterne la semi-vérité avec quelques silences, et s'ingénie à «retoucher » pratiquement toute la tragédie vendéenne, véritable tache noire sur la face de la Révolution :

La situation des révoltés vendéens était bien plus favorable que la position des armées républicaines. Connaissant parfaitement le terrain, les Vendéens se déplaçaient rapidement par petits détachements, sur les sentiers étroits, pratiquement invisibles, à travers la forêt, les collines et les marais. Ils attaquaient l'ennemi subitement, embusqués derrière les buissons, les taillis, les hauteurs, et ils disparaissaient tout aussi soudainement. Pour les républicains, les déplacements en territoires inconnus, en l'absence de bonnes routes, provoquaient des difficultés extraordinaires. Ils se déplaçaient par grandes colonnes, dans la mesure où se diviser en petits détachements les aurait conduits indubitablement à la mort. Ils avançaient lentement, avec hésitation, regardant autour d'eux à chaque instant, comme des bêtes traquées. Il ne pouvait être question de se reposer et de s'arrêter pour rassembler leurs forces. Bien évidemment, les républicains ne pouvaient pas non plus compter sur l'hospitalité des Vendéens. Tandis que les Vendéens révoltés pouvaient trouver partout des refuges et de la nourriture, les républicains étaient obligés de traîner avec eux leurs provisions pour ne pas mourir de faim. Tourmentés dans leurs corps et leurs âmes, ils faisaient retomber leur colère sur les Vendéens, qui leur rendaient bien la pareille. ${ }^{33}$ 
Dans les principes, il n'y a guère ici de quoi polémiquer. L'auteur est plus ou moins exact dans les détails, mais sous un déluge de paroles, il « ruse » en passant sous silence le principal. En effet, la tragédie vendéenne, ce ne sont pas les difficultés rencontrées par les troupes républicaines «tourmentées » dans leur progression, ni le fait qu'elles « faisaient retomber leur colère » sur leurs adversaires. La véritable tragédie, c'est bien le fait qu'en suivant les ordres du pouvoir révolutionnaire, ces troupes ont cherché l'extermination systématique et dirigée de la population civile, après la fin même de la phase active des hostilités militaires. Au contraire, l'auteur souligne avec force que les incidents qui se sont déroulés dans le département rebelle n'ont pas dépassé les limites habituelles des «coûts » de guerre, dans la mesure où «malgré toutes ces mesures sévères, la guerre intestine ne finissait toujours pas ». Enfin, en conclusion, ce même auteur termine par un happy end :

La paix ne fut finalement conclue avec les Vendéens, qu'en 1795. Pourtant, si l'on en juge par les conditions de cette paix, la victoire des républicains était loin d'être complète. Il est vrai que les Vendéens acceptèrent de reconnaître le gouvernement républicain. En revanche toutes leurs pertes furent compensées En outre, ils furent exemptés du service militaire, et leur liberté religieuse leur fut rendue. ${ }^{34}$

Toutefois l'auteur omet de dire, que cette paix n'a pas tenu six mois et que les « pertes » s'élevaient à $25 \%$ de la population de Vendée.

D'ailleurs, tous ces « frais de guerre » de la Révolution étaient mentionnés rapidement et soigneusement amoindris. Ils étaient surtout « rachetés », d'après les auteurs cités, par ses bilans glorieux, qui étaient exaltés dans les termes les plus enthousiastes :

La signification de la première Révolution est immense, et pas uniquement en France, mais pour toute l'humanité. Les messages qu'elle a légués de la liberté, de l'égalité et de la fraternité se répandirent sur toute la terre comme une nouvelle foi. 35

Le peuple s'est réveillé d'un sommeil séculaire, a redressé son dos voûté, a tendu ses muscles. Le vieil édifice a tremblé, la violence a fait craquer toutes les coutures, et il s'est écroulé sous les acclamations du peuple triomphant. ${ }^{36}$

Le culte de la Révolution française diffusé parmi les élites intellectuelles de la Russie est un des facteurs importants, qui a conditionné le soutien enflammé de larges couches de l'intelligentsia en faveur du renversement de la monarchie. Selon la vision romantique de la «révolution festive », qui leur était bien connue, ces intellectuels attendaient que la révolution tourne en fête en Russie aussi. Ces sentiments sont brillamment décrits par V.P. Kataev dans une nouvelle aux nombreux traits autobiographiques : « À cette époque légendaire, la jeunesse était habituée, en

34. Ibid., p. 90.

35. Olenina, Vesna narodov..., p. 79.

36. Nikolin, Andreev, Velikij perevorot..., $1^{\text {re }}$ partie, p. 4. 
quelque sorte, à jouer un peu à la Révolution française, tutoyant tout le monde, appelant chacun "citoyen" ou "citoyenne", comme si le monde nouvellement né de la révolution russe était fait de Saint-Justs, Dantons, Desmoulins, Marats et Robespierres ${ }^{37}$. Cependant tous les personnages de cette nouvelle ne «jouent» pas à la Révolution française, en essayant sur eux-mêmes les modèles de ses divers protagonistes. Le héros principal, un jeune homme d'une famille d'intellectuels, est lui aussi séduit par le «romantisme de la révolution », voyant dans la révolution russe le reflet attirant d'une autre révolution, celle de France, qui lui est connue depuis l'enfance : «...la Convention... le Palais Royal... la branche verte de Desmoulins. Ça ira !... et soudain s'empare de lui sa passion pour une jeune fille du peuple, en laquelle il voit Théroigne de Méricourt, menant derrière elle les foules de sans-culottes. Le bonnet phrygien et le profil classique $»^{38}$. Plus les espérances sont sublimes, plus la déception est amère. La «fête » attendue tourne en tragédie, et ce n'est pas le Pont-neuf de Paris qui se présente devant le jeune homme, mais le pont Sabaneevskij d'Odessa, le chemin vers la cave aux exécutions de la Tcheka.

En décrivant l'incompréhension et la peur, la sensation d'une tromperie monstrueuse, voire même de sa propre illusion, V.P. Kataev n'exagère pas du tout. Ces mêmes sentiments se retrouvent dans les œuvres des publicistes de l'époque, qui appartenaient aux dirigeants des élites intellectuelles. « Nous nous sommes trop dépêchés en qualifiant notre révolution de grande, et nous l'avons comparée à la Grande Révolution française », écrivait Fedor Sologub dans son essai Le baptême de la saleté (1918). « Mais maintenant nous voyons, que de nos jours il y a peu de grandeur, et que notre révolution n'est qu'un singe de la Grande Révolution française. [...] Celle-ci, réellement grande, était toute entière dirigée par l'amour pour la France, pour la patrie, et le révolutionnaire se sentait avant tout un patriote. Eh bien, chez nous, évidemment, tout est le contraire. [...] De feu et de sang était ce baptême, apporté à l'Europe par la France révoltée contre le despotisme. Le diable abject qui a pris possession de nous, rit violemment aux éclats, et nous couvre de boue... $»^{39}$.

Léonid Andreev était tellement frappé par les événements russes de 1917, et leur non-conformité à cette révolution si longtemps attendue, qu'il leur a refusé le droit même d'être désignés comme tels, et les a qualifiés de révolte (bunt). Il a opposé les notions de «mauvaise » révolte russe et de «bonne» révolution, celle-ci étant définie de toute évidence par les traits familiers de la vision idéale de la Révolution française. «Les slogans de la révolution sont toujours universels. Pour elle, comme

37. Valentin P. Kataev, Uže napisan Verter, M.: Panorama, 1992, p. 374.

38. Ibid., p. 338-339. Cf. «Qui de nous n'a pas écrit alors avec ravissement au sujet de la cocarde verte de Desmoulins, dans ces jours où le lycéen Kannegisser tirait sur Urickij, et Kaplan, avec une balle empoisonnée, sur Lénine. Ce n'étaient pas les sans-culottes avec leurs bonnets phrygiens rouges, qui portaient sur des piques les têtes des aristocrates, mais les ouvriers de l'usine Putilov, en vieilles vestes et casquettes, ceints de bandes mitrailleuses, qui protégeaient Smol’nyj », in V.P. Kataev, Almaznyj moj venec, M., 1979. p. 207-208.

39. «Dans les jours de la création de l'enfer... (Iz publicistiki F. Sologuba 1918 g.) », Russkaja mysl’ [La pensée russe], $\mathrm{n}^{\circ}$ 4145. 17-23 octobre1996, p. 11. 
pour Dieu, toute personne compte. Telle la Justice incarnée, elle protège les droits de l'être humain. [...] Liberté, égalité et fraternité. Voici la loi inébranlable de la Révolution... $»^{40}$. Ne parvenant pas à comprendre pourquoi, à la place de la « merveilleuse » révolution, s'est produite cette révolte monstrueuse, Andreev, tout comme Sologub, accuse aussi « le diable, vivant en nous ». C'est lui qui « a aveuglé et a tout embrouillé, a mélangé les cartes, a transformé tous les slogans en bouillie sans nom et a marié dans une union contre-nature, une victime, la révolution, et son meurtrier, cette absurde, stérile et sanglante révolte russe $»^{41}$.

La révolution de 1917 a détruit le «mythe russe » de la Révolution française. L'idée qu'il n'y a jamais eu de «bonne » révolution, même en France, trouve son expression dans la littérature des émigrés, à travers tous les genres ${ }^{42}$. Par exemple, dans ces vers d'Ivan Savin :

Tout cela a déjà été. Il n'y a qu'une voie

Pour la populace actuelle et ancienne.

Seule différence : les ombres de nos guillotines,

Sont plus longues et plus rebelles.

Le poète adresse aussi ses reproches aux créateurs du mythe de la Révolution française :

Tout le sang des places de Paris,

Des pierres et des mains, la légende l'a effacé... ${ }^{43}$

Cependant il n'était possible de parler ainsi qu'à l'étranger. En Russie, à la place de l'ancien « mythe russe », protégé par l'opinion de l'intelligentsia «progressiste », un nouveau point avait vu le jour, le «mythe soviétique» de la Révolution française, prôné désormais avec toute la puissance de l'idéologie d'État et de l'appareil répressif.

(traduit du russe par Julie Ollivier)

Institut d'histoire universelle, Académie des sciences de Russie

Centre d'études du XVIII' siècle

tchoudin@mail.ru

40. Leonid N. Andreev, «Evropa v opasnosti », in L.N. Andreev, Pered zadačami vremeni, Benson: Chalidze Publ., 1985, p. 201.

41. Ibid., p. 201.

42. Voir, par exemple, Petr Struve, Razmyšlenija o russkoj revoljucii, Sofia : Rossijskobolgarsk knigoizdat, 1921, ou encore Aleksej Aleksandrovič Gol'denveizer, Jakobincy i bol`̌̌seviki, Berlin, 1922.

43. I. Savin, «Ladonka [1925] », dans Russkaja mysl’ [La pensée russe], 1996. n 4147, 31 octobre- 6 novembre. 